\title{
Prenatal diagnosis of Pfeiffer syndrome type 2 with increased nuchal translucency
}

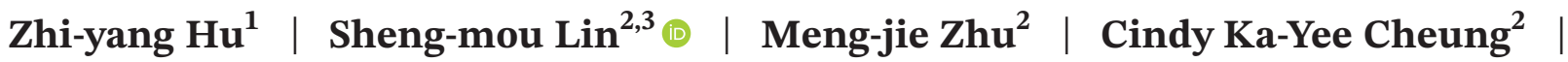 \\ Tao Liu ${ }^{4}$ Jin $\mathrm{Zhu}^{5}$ \\ ${ }^{1}$ Department of Obstetrics, Shenzhen People's Hospital, Shenzhen, China \\ ${ }^{2}$ Department of Obstetrics and Gynecology, The University of Hong Kong - Shenzhen Hospital, Shenzhen, China \\ ${ }^{3}$ The First School of Clinical Medicine, Southern Medical University, Guangzhou, China \\ ${ }^{4}$ Department of Ultrasound, Shenzhen People's Hospital, Shenzhen, China \\ ${ }^{5}$ Department of Radiology, Shenzhen People's Hospital, Shenzhen, China
}

\section{Correspondence}

Sheng-mou Lin, Department of Obstetrics and Gynecology, The University of Hong Kong - Shenzhen Hospital, Haiyuan 1st road, Futian District, Shenzhen, China.

Email:linsm@hku-szh.org

\section{Funding information}

Shenzhen Municipal Committee of Science and Technology Innovation, Shenzhen, China, Grant/Award Number: JCYJ20170307171743182. High Level-Hospital Program, Health Commission of Guangdong Province, P. R. China, Grant/Award Number: HKUSZH201902017

\begin{abstract}
Pfeiffer syndrome (PS) is a rare autosomal dominant genetic disorder characterized by craniosynostosis, broad thumbs / toes. Here, we report a case of PS type 2 with increased nuchal translucency in early trimester.
\end{abstract}

\section{K E Y W O R D S}

increased nuchal translucency, Pfeiffer syndrome, prenatal diagnosis

\section{1 | INTRODUCTION}

Pfeiffer syndrome (PS, OMIM \#101600) is a rare autosomal dominant genetic disorder characterized by craniosynostosis, broad thumbs / toes with an incidence of 1/100,000 live birth. ${ }^{1}$ There are three clinical subtypes. ${ }^{2}$ Type 1 is associated with midface hypoplasia, broad thumbs, great toes, and is compatible with life, with normal intelligence. Type 2 is characterized by cloverleaf skull, severe ocular proptosis, elbow ankyloses, and large halluces and thumbs. Type 3 is similar to type 2 except for cloverleaf skull, but with visceral malformation. Fetuses with type 2 or type 3 usually die in utero or in early infancy. With development of ultrasound technology and application of 3-D ultrasound examination, prenatal diagnosis of Pfeiffer syndrome has been reported since $1996 .{ }^{3}$ However, craniosynostosis, limb, and visceral malformation are mostly be detected in the second or third trimester. Little is known about the ultrasound manifestation of Pfeiffer syndrome in early pregnancy. Although increased NT has been observed as indirect fetal signs of syndromic or non-syndromic craniosynostosis at first-trimester 
ultrasound examination, Pfeiffer syndrome with increased NT has not been reported so far. ${ }^{4,5}$ Here, we report a case of Pfeiffer syndrome type 2 with increased nuchal translucency in first trimester.

\section{CASE REPORT}

A healthy 30-year-old nulliparous woman underwent first-trimester fetal ultrasound scan at $12^{+1}$ weeks' gestation, which showed a single fetus with an increased nuchal translucency (NT) of $3.1 \mathrm{~mm}$ (> 99th centile) and a crown-rump-length of $51 \mathrm{~mm}$ (Figure 1A). Her husband was 41 years old and healthy. The couple was nonconsanguineous. There was no family history of congenital anomalies. Non-invasive prenatal test (NIPT) at 16 weeks' gestation showed low-risk for fetal Down syndrome.

Morphologic scan at 22 weeks showed acrocephaly, temporal indentation, prominent lateral ventricle with anteroposterior diameter of the posterior horn measuring $10 \mathrm{~mm}$, lordosis of the thoracic spine, and broad thumbs and great toes (Figures 1B,C and 2). The fetal sagittal suture was narrow. Its coronal and lambdoid sutures were nearly closed whereas the metopic suture was wide. Pfeiffer syndrome type 2 was suspected based on the typical ultrasound findings. Cordocentesis was performed for molecular diagnosis followed by parents decided to terminate the pregnancy. A $420 \mathrm{~g}$ female abortus was delivered. Examination revealed cloverleaf head, proptosis, hypertelorism, low-set ears, flat nasal bridge, abducted broad

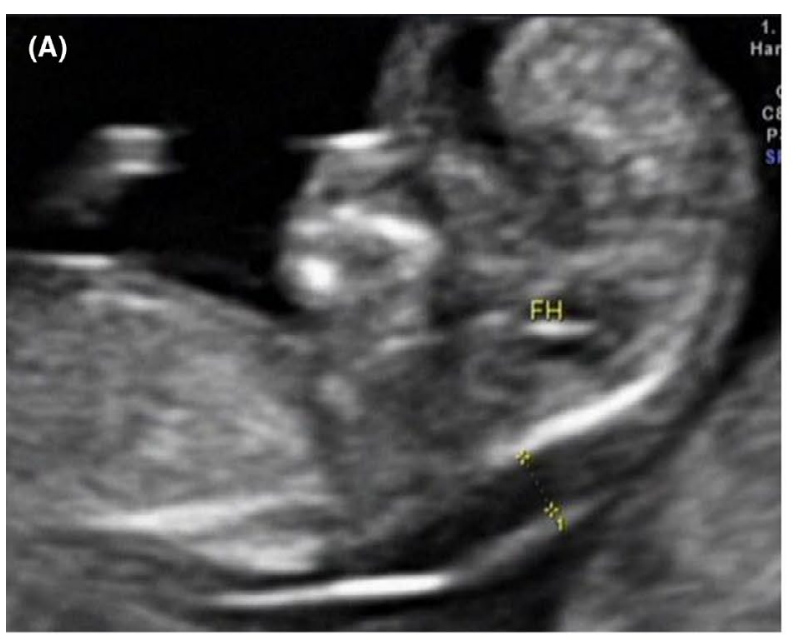

(B)

(C)
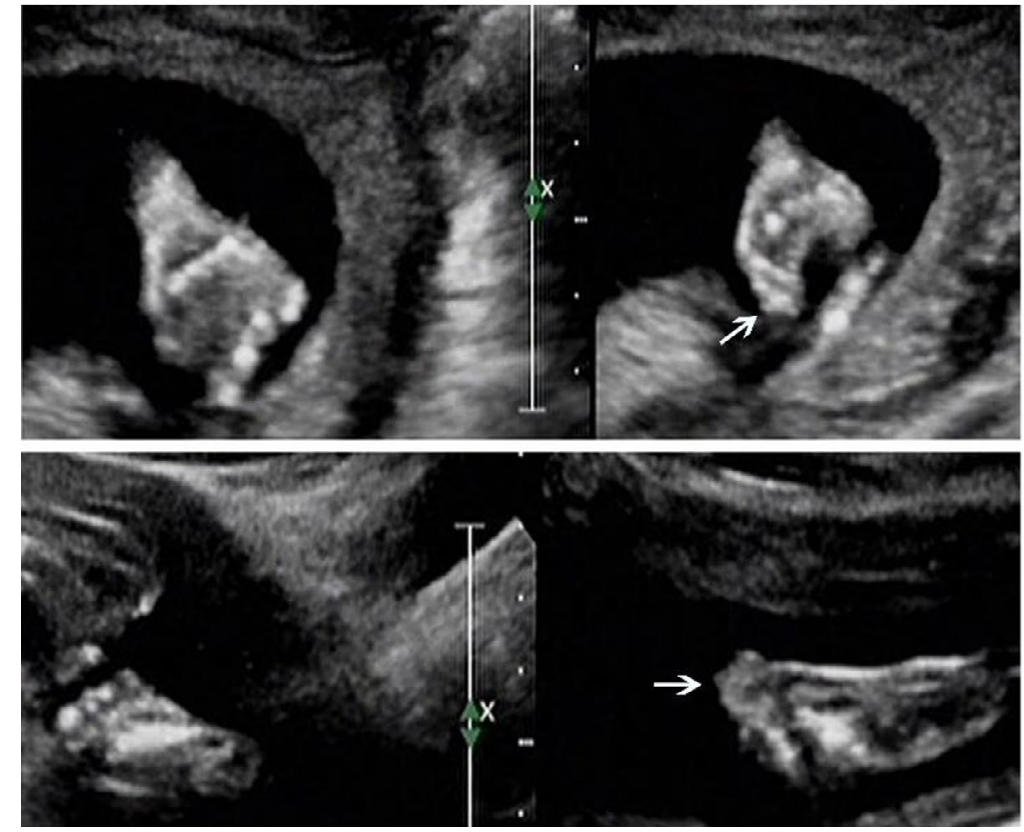

F I G U RE 1 (A) Increased nuchal translucency of the fetus at $12^{+1}$ weeks of gestation. (B) Acrocephaly, protruding forehead, bilateral temporal indentation of the skull and mild ventriculomegaly. (C) Broad thumbs and great toes found by prenatal ultrasound 

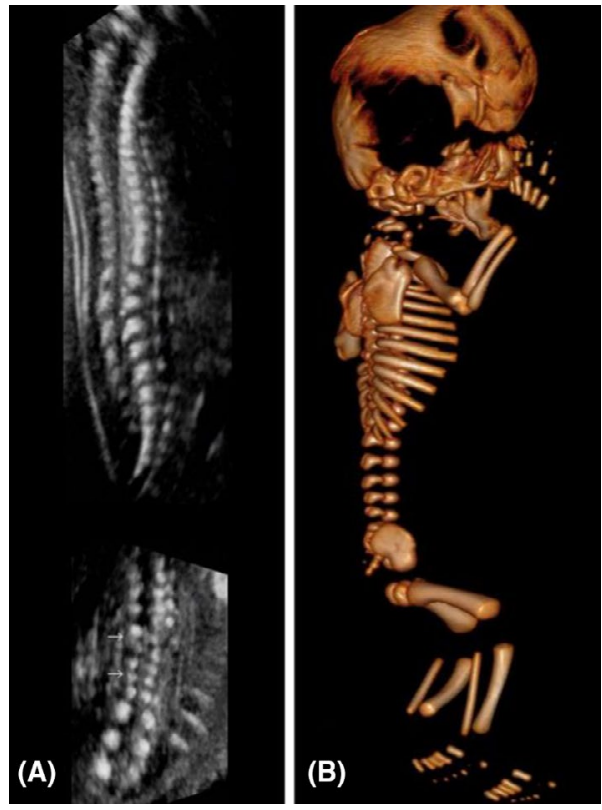

FI G URE 2 Thoracic spine lordosis and narrow, vertebral fusion, sacrococcygeal eversion found by prenatal ultrasound (A) and postnatal 3D CT (B) thumbs, and abducted broad toes along with overriding toes bilaterally (Figure 3). Sacrococcygeal eversion was noted by 3D computed tomography (CT) scan, which was consistent with prenatal ultrasound pictures in retrospect (Figure 2). Whole-exome sequencing showed a heterozygous pathogenic variants on FGFR2 gene [c.870G $>$ T] (located at exon 7), predicted to encode a Trp290Cys substitution. Parental FGFR2 sequencing showed normal findings; therefore, the fetal mutation was de novo.

\section{DISCUSSION}

There are a number of genetic syndromes with craniosynostosis, such as Apert syndrome, PS, Crouzon's disease, and Saethre-Chotzen syndrome. With the low incidence and the wide variability of morphological findings, prenatal diagnosis of Pfeiffer syndrome might be challenging. To our knowledge, more than 30 prenatally diagnosed PS have been reported, ${ }^{6-8}$ all were diagnosed at or beyond 20 weeks of gestation. Although cloverleaf skull was one of the most common characteristic noted
FIGURE 3 Postmortem examination. (A) Frontal view, showing the typical cloverleaf head, hyertelorism, and contractures of multiple joints. (B) Lateral view, showing the low-set posteriorly rotated ear, midface hypoplasia, proptosis. (C-D) Abducted broad thumb. (E) Broad great toes of feet and overriding toes of the left foot
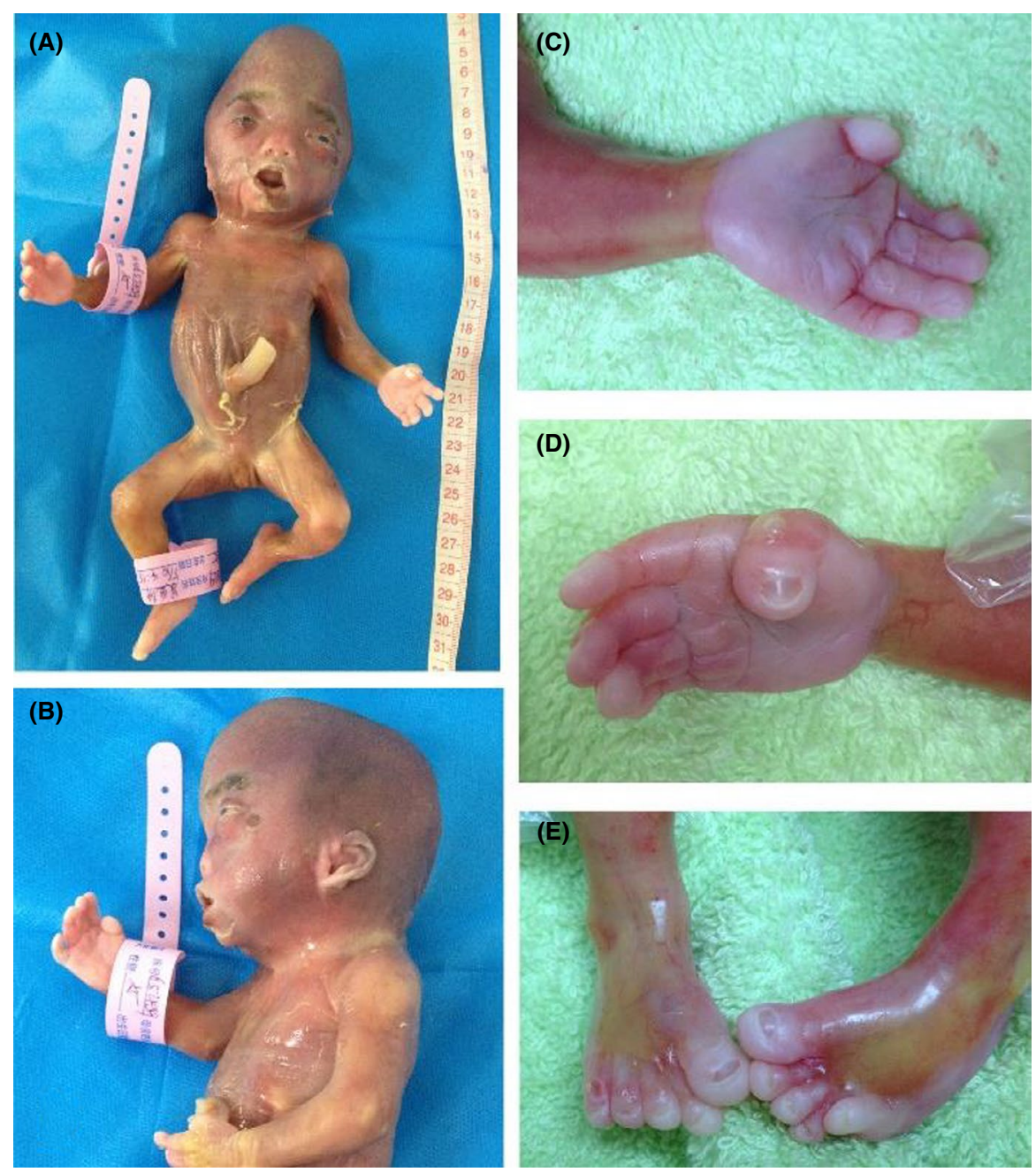
in fetal period, ${ }^{7}$ the typical cloverleaf skull might unlikely be detectable prior to 20 weeks. ${ }^{7,9,10}$ Gomez-Gomez revealed strawberry-shaped cranium, hypertolerism, a supernumerary bone at frontal level, small thorax, kyphosis and dorsal level scoliosis, and suspicious bladder exstrophy at 20 weeks of gestation as clues for the diagnosis. ${ }^{9}$ Nazzaro detected bilateral temporal indentation and hypertelorism at 20 weeks. ${ }^{10}$ Gorincour has reported fetus with PS presenting with a thickened nuchal fold and choroid plexus cysts at 20 weeks' gestation, frontal bossing and temporal indentation at 23 weeks' gestation, and typical cloverleaf skull, broad thumbs and slight hypertelorism at 24.5 weeks' gestation. ${ }^{11}$ The relatively late presentation of cloverleaf head might be a result of rapid growth of the brain in the late half pregnancy and craniosynostosis. Among the prenatal ultrasound features, abnormal fetal skull shape $(72.2 \%)$ was most frequently reported, while proptosis and hypertelorism were noted in $44.4 \%$ cases, whereas malformation of thumbs and toes were found prenatally in $33.3 \%$ and $38.9 \%{ }^{6}$ Ventriculomegaly may be a helpful clue in detecting craniosynostosis when fetal skull deformation is minimal, ${ }^{7}$ which presented in our case. Presence of a sacral appendage and vertebral fusion is also suggestive of $\mathrm{PS},{ }^{12}$ which also detected in this case.

The ultrasound presentation of PS type 2 in the first trimester has not been described before. In our case, the first abnormal ultrasound appearance was increased nuchal translucency, which might be the first detectable sign in severe craniosynostosis. In fact, increased nuchal translucency has been reported in a case with Apert syndrome, which is also a craniosynostosis syndrome with FGFR2 mutation. ${ }^{13}$ Thickened nuchal fold (NF) and cystic hygroma with PS were reported presented at 20 weeks and 16 weeks, respectively. ${ }^{7,11}$ Thickened NF or increased NT is a result of abnormal accumulation of lymph fluid, which might be due to abnormality of blood and lymphatic vessels. In syndromic craniosynostosis, the skull base is smaller and the stenotic jugular foramen causes the crowding of the posterior fossa. Consequently, venous pressure rises and higher cerebrospinal fluid pressure is required to maintain balance, which might explain the severe cases who developed increased NT and ventriculomegaly. ${ }^{14}$

Another possible association involves molecular mechanisms; fibroblast growth factors (FGFs) are consisted of a family of nine heparin-binding polypeptides which enroll cell proliferation, differentiation, and migration. Any alteration of FGFR can have influence on cellular response to FGFs. FGF/FGFR genes play a key role in complex branched structures development, such as tracheal bifurcation and lung system, ${ }^{15}$ limb,${ }^{16}$ cranial sutures, ${ }^{17}$ and angiogenesis. ${ }^{18,19}$ Mutation in FGFR2 can cause abnormal angiogenesis of fetus, which might also explain the increased NT in this case and David's case. ${ }^{13}$

PS mutations have been reported in the ligand-binding region of both FGFR1 and FGFR2. However, mutations affecting the FGFR2 have been reported not only in PS cases but also in other syndrome with craniosynostosis including Crouzon, Apert, and Jackson-Weiss syndromes. ${ }^{20}$ Combined with our case, 16 of 19 prenatal diagnosed cases of PS with genetic test were found to have mutations in FGFR2 (78.5\%), and seven of them were Try290Cys substitution $(43.8 \%)^{6,8-10,12,21}$

The correlation between Trp290Cys substitution in FGFR2 and PS has been reported since $1997 .^{10,21-24}$ The codon 290 exon 7 of FGFR2 is characterized by immunoglobulin-like hoops formed by cys crosslinking, whereas an additional cys at the site caused by a Trp290Cys substitution as our case forms abnormal crosslinking, and changes 3D structure of FGFR2. ${ }^{20}$ Although cases with such mutations can have variable phenotypes presenting as PS type 2 or type $3,^{10,21,22,25}$ their clinical manifestations are always severe as syndromic craniosynostosis.

\section{ACKNOWLEDGEMENT}

This work has been supported by High Level-Hospital Program, Health Commission of Guangdong Province (HKU-SZH201902017), P. R. China and research grants JCYJ20170307171743182 from the Shenzhen Municipal Committee of Science and Technology Innovation, Shenzhen, China.

\section{CONFLICTS OF INTEREST}

There is no any conflict of interest in relation to the work.

\section{AUTHOR CONTRIBUTIONS}

Zhi-yang Hu and Sheng Mou Lin involved in conceptualization, methodology, drafting, and writing manuscript. Meng-jie Zhu involved in conceptualization, reviewing, and editing. Cindy Ka-Yee CHEUNG, Tao Liu, and Hongtao Jin involved in data collection and curation.

\section{CONSENT}

Appropriate consent has been obtained from patient, prior to submission, in regards of the publication of images and data.

\section{DATA AVAILABILITY STATEMENT}

All data presented and analyzed in this report are included in the published article.

\section{ORCID}

Sheng-mou Lin (1) https://orcid.org/0000-0001-9033-3471 


\section{REFERENCES}

1. Vogels A, Fryns JP. Pfeiffer syndrome. Orphanet J Rare Dis. 2006;1:19.

2. Cohen MM Jr. Pfeiffer syndrome update, clinical subtypes, and guidelines for differential diagnosis. Am J Med Genet. 1993;45(3):300-307.

3. Bernstein PS, Gross SJ, Cohen DJ, et al. Prenatal diagnosis of type 2 Pfeiffer syndrome. Ultrasound Obstet Gynecol. 1996;8(6):425-428.

4. Syngelaki A, Hammami A, Bower S, et al. Diagnosis of fetal non-chromosomal abnormalities on routine ultrasound examination at 11-13 weeks' gestation. Ultrasound Obstet Gynecol. 2019;54(4):468-476.

5. Dall'Asta A, Paramasivam G, Lees C, et al. The brain shadowing sign: A clue finding for early suspicion of craniosynostosis? Fetal Diagn Ther. 2019;45(5):357-360.

6. Giancotti A, D’Ambrosio V, Marchionni E, et al. Pfeiffer syndrome: Literature review of prenatal sonographic findings and genetic diagnosis. J Matern Fetal Neonatal Med. 2017;30(18):2225-2231.

7. Harada A, Miyashita S, Nagai R, et al. Prenatal sonographic findings and prognosis of craniosynostosis diagnosed during the fetal and neonatal periods. Congenit Anom (Kyoto). 2019;59(4):132-141.

8. Torres-Canchala L, Castaño D, Silva N, et al. Prenatal diagnosis of Pfeiffer syndrome patient with FGFR2 C.940-1G>C variant: A case report. Appl Clin Genet. 2020;13:147-150.

9. Gómez-Gómez JL, Fernández-Alonso AM, Moreno-Ortega I, et al. Prenatal diagnosis of Pfeiffer syndrome prior to 20 weeks' gestation. J Obstet Gynaecol. 2013;33(3):309-310.

10. Nazzaro A, Della Monica M, Lonardo F, et al. Prenatal ultrasound diagnosis of a case of Pfeiffer syndrome without cloverleaf skull and review of the literature. Prenat Diagn. 2004;24(11):918-922.

11. Gorincour G, Rypens F, Grignon A, et al. Prenatal diagnosis of cloverleaf skull: watch the hands! Fetal Diagn Ther. 2005;20(4):296-300.

12. Saliba S, Morel B, Gonzales M, et al. Variable prenatal presentation of Pfeiffer syndrome: Suggested aids to prenatal sonographic diagnosis. Prenat Diagn. 2018;38(5):349-356.

13. David AL, Turnbull C, Scott R, et al. Diagnosis of Apert syndrome in the second-trimester using $2 \mathrm{D}$ and $3 \mathrm{D}$ ultrasound. Prenat Diagn. 2007;27(7):629-632.

14. Rijken BF, Leemans A, Lucas Y, et al. Diffusion tensor imaging and fiber tractography in children with craniosynostosis syndromes. AJNR Am J Neuroradiol. 2015;36(8):1558-1564.
15. Chen CP, Lin SP, Su YN, et al. Craniosynostosis and congenital tracheal anomalies in an infant with Pfeiffer syndrome carrying the W290C FGFR2 mutation. Genet Couns. 2008;19(2):165-172.

16. Horakova D, Cela P, Krejci P, et al. Effect of FGFR inhibitors on chicken limb development. Dev Growth Differ. 2014;56(8):555-572.

17. Cohen MM Jr. Perspectives on craniosynostosis: sutural biology, some well-known syndromes, and some unusual syndromes. $J$ Craniofac Surg. 2009;20(Suppl 1):646-651.

18. Robson CD, Mulliken JB, Robertson RL, et al. Prominent basal emissary foramina in syndromic craniosynostosis: correlation with phenotypic and molecular diagnoses. AJNR Am J Neuroradiol. 2000;21(9):1707-1717.

19. Dienstmann R, Rodon J, Prat A, et al. Genomic aberrations in the FGFR pathway: opportunities for targeted therapies in solid tumors. Ann Oncol. 2014;25(3):552-563.

20. Schaefer F, Anderson C, Can B, et al. Novel mutation in the FGFR2 gene at the same codon as the Crouzon syndrome mutations in a severe Pfeiffer syndrome type 2 case. Am J Med Genet. 1998;75(3):252-255.

21. Ariga H, Endo Y, Ujiie N, et al. Trp290Cys mutation of the FGFR2 gene in a patient with severe Pfeiffer syndrome type 2 . Pediatr Int. 2001;43(3):293-295.

22. Tartaglia M, Valeri S, Velardi F, et al. Trp290Cys mutation in exon IIIa of the fibroblast growth factor receptor 2 (FGFR2) gene is associated with Pfeiffer syndrome. Hum Genet. 1997;99(5):602-606.

23. Barry GP, Ny BM, Zackai EH, et al. A case report of a patient with Pfeiffer syndrome, an FGRF 2 mutation (Trp290Cys) and unique ocular anterior segment findings. Ophthalmic Genet. 2010;31(4):193-195.

24. Oliveira NA, Alonso LG, Fanganiello RD, et al. Further evidence of association between mutations in FGFR2 and syndromic craniosynostosis with sacrococcygeal eversion. Birth Defects Res A Clin Mol Teratol. 2006;76(8):629-633.

25. Ettinger N, Williams M, Phillips JA 3rd. Variable expressivity and clinical heterogeneity can complicate the diagnosis and management of Pfeiffer syndrome. J Craniofac Surg. 2013;24(5):1829-1832.

\section{How to cite this article: Hu Z-Y, Lin S-M,} Zhu M-J, Cheung CK-Y, Liu T, Zhu J. Prenatal diagnosis of Pfeiffer syndrome type 2 with increased nuchal translucency. Clin Case Rep. 2021;9:e05001. https://doi.org/10.1002/ccr3.5001 